\title{
E19 ON APPROXIMATION OF PHASE EQUILIBRIA OF MULTICOMPONENT HYDROCARBON MIXTURES AND PREDICTION OF OIL DISPLACEMENT BY GAS INJECTION
}

\author{
V.M. ENTOV ${ }^{1,2}$, F.D.TURETSKAYA ${ }^{1}$, D.V. VOSKOV ${ }^{1}$ \\ 1) Institute for Problems in Mechanics, Russian Academy of Sciences \\ prospekt Vernadskogo, 101, korp.1, 119526, Moscow, RUSSIA \\ e-mail: entov@ipmnet.ru \\ 2) Gubkin Russian State Oil and Gas University.
}

\begin{abstract}
This study is direct continuation of that presented in [1-7]. Further progress in using splitting of the problem of multicomponent two-phase flow into physicochemical and hydrodynamical parts is reported. First, conditions of phase equilibrium are expressed in terms of new variables, namely tie-line parameter and concentration of a component. An effective procedure for such transformation is described. Next, it is shown, that for $1 \mathrm{D}$ displacement the property of route invariance holds, namely projection of the solution into the tie-line parameter space remains the same for different fluid-dynamical parameters, even in the case of composition-dependent density. It is demonstrated also, that the property holds approximately also for more general flows, including 2D flows in homogeneous and non-homogeneous reservoirs. Some examples are presented for oil displacement by injection of $\mathrm{N}_{2}$ or $\mathrm{CO}_{2}$ - containing gas.
\end{abstract}

1. Alternative description of phase equilibria. It was shown previously, that the problem of oil by gas injection ("miscible gas flooding") in 1D statement can be splitted into two successively solved problems, namely a physicochemical and hydrodynamical ones, so that the physicochemical problem is expressed entirely in terms of phase equilibria (see [1-7]). A key element in such a splitting consists in transformation of the conditions of phase equilibria to new variables, namely concentration of a ("leading") component $C$, and a vector-parameter $\gamma$ of tie lines.

We consider a system of $n$ components that may form a single-phase or two-phase mixture. Composition of a system is specified by the composition vector $\mathbf{C}=\left\{C_{1}, \ldots C_{n_{C}}\right\}$. We use molar concentrations $C_{i}$ to characterize amount of given component in the mixture; overall and partial molar densities are denoted by $N$ and $N_{i}$, correspondingly. The concentrations satisfy the obvious relation

$$
C_{1}+\ldots+C_{n}=1 .
$$

Therefore, composition vector can be represented by a point within $(n-1)$ - dimensional regular polyhedron of unit height (a triangle for $n=3$, a tetrahedron for $n=4$, etc).

The interior of the polyhedron is divided into single-phase and two-phase domains separated with a $\left(n_{C}-2\right)$ dimensional binodal surface, the locus of bubble-points and dew-points at given values of pressure and temperature. Thermodynamics implies that there is one-to-one correspondence between the sets of bubble points and due points. This correspondence is specified by the requirement that compositions corresponding to two respective points are in thermodynamic equilibrium. Let $\mathbf{C}^{l}$ and $\mathbf{C}^{g}$ be two such points. Then all compositions along the straightline segment $\theta$ :

$$
\theta: \mathbf{C}=S \mathbf{C}^{l}+(1-S) \mathbf{C}^{g} ; \quad 0 \leq S \leq 1 .
$$

correspond to two-phase mixtures of liquid volume fraction $S$ of composition $\mathbf{C}^{l}$ and gas volume fraction $(1-S)$ of composition $\mathbf{C}^{g}$; we will refer to $S$ as the "liquid phase saturation", or just "saturation". The segment $\theta$ is a tie-line.

Equations (2) for $S<0$, or $S>1$, define tie-line extensions beyond the two-phase domain. Two tie-lines do not intersect within the compositional space, as given composition breaks into a unique combination of the liquid and gaseous phases. Therefore, there exists one-to-one corre- 


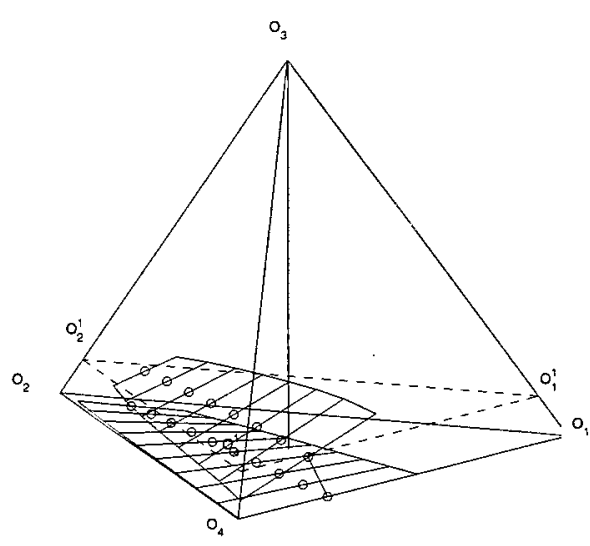

Fig.1 Scanning the composition space to get basis set of tie-lines.

spondence between any two of three sets: the set $B$ of the bubble points, the set $\Delta$ of dew points, and the set $\Theta$ of tie lines: $B \leftarrow \Delta \leftarrow \rightarrow \Theta$. All compositions covered by tie-lines and their extensions form a subspace $E$ in composition space. Geometry of tie-lines defines an algebraic structure, such that each point ("composition") within $E$ is specified with a tie line and position of the composition point along the tie line (specified with, say, liquid phase fraction $S$, or another parameter, such as concentration of one of components $C$, along the tie line).

The main idea is to use these alternative coordinates in the composition space as main variables. Intuitively, we expect that the natural coordinate system will eventually lead to some advantages in description of multicomponent flow. This idea assumes a more definite shape after analysis of some key problems of the theory of gas injection for EOR (see [1-7]).

2. Phase equilibrium in natural coordinates. Here, we first describe a technique developed to transform the conditions of phase equilibrium to alternative coordinates. Notice, that there is no obvious preferred way to introduce the alternative coordinates. Therefore, the approach relies upon simple observations of "typical" behavior predicted by standard software based upon EOS flash calculation.

First, a number of tie-lines is generated. To this end, for a number of points within the twophase domain the standard flash calculations are performed using a EOS-code (specifically, the ECLIPSE PVTi package was used throughout this study).

The points are chosen using a scanning procedure illustrated in Fig.1.

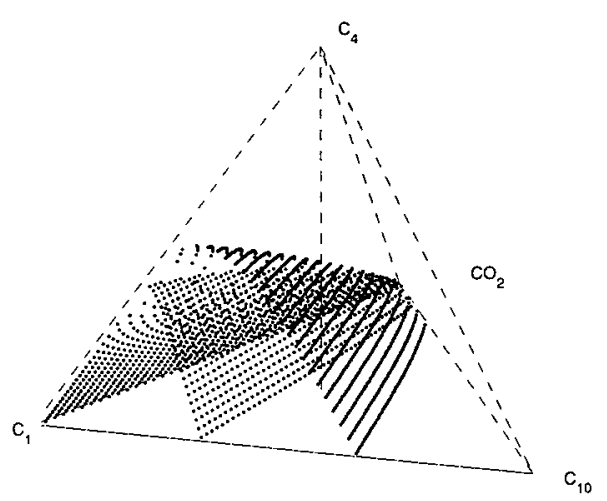

Fig.2 Dew-point, bubble-point and median surfaces the system $\left[\mathrm{CO}_{2}, \mathrm{C}_{1}, \mathrm{C}_{4}, \mathrm{C}_{10}\right]$ at $\mathrm{T}=345^{\circ} \mathrm{K}, \mathrm{P}=120$ Bars.

Its basic idea is to start from the midpoint of the longest tie-line at the edge of phase diagram, and then proceed layer-by-layer choosing each next computational point well within twophase domain of the compositional space. Such procedure proved to be robust for not-too-large number of components. Let $\mathbf{C}_{(j)}$ be a computational point. Then flash calculations result in respective values of $\mathrm{C}_{(j)}^{l}, \mathrm{C}_{(j)}^{g}, S$; respective phase densities and viscosities can also be calculated. Now we are in position to draw a number of tielines connecting points $\mathbf{C}_{(j)}^{l}, \mathbf{C}_{(j)}^{l}$. They form a basic set of tie lines. In fact, it is this discrete set of tie line that further is used as representing the entire set of tie lines.

There is no single obvious way to parameterize tie lines. However,observations of numerous 3- and 4- component phase diagrams suggest that two common properties approximately hold for the diagrams:

(1) The binodal surface has "parabola-like" (in 2d) or "paraboloid-like" (in 3d) shape.

(2) Midpoints of tie-lines are located along an "almost straight" line (in 2d) or along an "almost plane" median surface (in 3d) (see Fig.2).

Therefore, it is reasonable to take the values of $q=n-2$ concentrations of the components corresponding to the midpoints of tie-lines as components of the tie-line vector-parameter, and use one of two remaining concentrations as additional independent coordinate along tie-line. More precisely, we choose one of components (say, the most light one) as the leading or principal component, its concentration is used as the alongtie-line coordinate $C$; one of components (say, the heaviest one) is chosen as dependent one. 
Then all other components are referred to as intermediary. Let the midpoints of tie lines be specified by the composition $n$-component vectors $\mathbf{C}_{j}^{m}=\left\{C_{j 1}^{m}, C_{j 2}^{m}, \ldots, C_{j n}^{m}\right\}$. Then we introduce tie-line parameter vector $\gamma$ by the relation

$$
\gamma=\left\{C_{2}^{m}, \ldots, C_{n-1}^{m}\right\}
$$

Say, in the case of 4-component mixture concentrations the first component is used as leading one, and its concentration is denoted by $C$, while concentrations of the second and third components at midpoints of tie lines $C_{2}^{m}$ and $C_{3}^{m}$ are used as components $\gamma_{1}$ and $\gamma_{2}$ of the tie-line vector parameter $\gamma$. With such a choice, the midpoints fill a $q$-dimensional domain $\Delta_{\gamma}$ in $\gamma$ space contained within the domain

$$
\begin{gathered}
0 \leq \gamma_{k}, \quad k=1, \ldots, q \\
\gamma_{1}+\gamma_{2}+\ldots+\gamma_{q} \leq 1
\end{gathered}
$$

Boundary of the parameter domain consists of a number of parts, corresponding to $\gamma_{k}=$ $0, \quad k=1, \ldots, q$, and may include part $\partial \gamma_{c r}$ with $\gamma_{k}>0$ for all $k$, corresponding to critical points, i.e. points for which the bubble and dew points coincide. Notice, that this part is a $(q-1)$-dimensional manifold.

Specification of the $\gamma$-domain $\Delta \gamma$ and of two vector functions $\mathbf{C}^{l}(\boldsymbol{\gamma})$ and $\mathbf{C}^{g}(\boldsymbol{\gamma})$ on this domain defines completely two-phase boundary in the composition space and pairwise correspondence between dew points and bubble points.

In fact, we know only a finite number of points in $\Delta_{\boldsymbol{\gamma}}$ and respective bubble and dew points:

$$
\gamma_{j} \rightarrow C_{j}^{l} ; \quad \gamma_{j} \rightarrow C_{j}^{g}
$$

In order to complete transformation to new coordinates in composition space, we have to describe respective dependencies throughout $\Delta_{\boldsymbol{\gamma}}$. It can be done in many different ways using different approximation or interpolation techniques. In this study, we used simple analytic expressions for $C^{l}$ and $C^{g}$ as functions of $\gamma$ suggested by the observed shape of the two-phase domain. Namely, the two-phase boundary has been approximated as follows.

First, the phase concentrations of the intermediary components are approximated as

$$
\begin{gathered}
C_{i}^{l}=\gamma_{i-1} P_{i}^{(2)}(\gamma) \\
C_{i}^{g}=2 \gamma_{i-1}-C_{i}^{l} ; i=2, \ldots, n-1,
\end{gathered}
$$

$P_{i}^{(2)}(\gamma)$ being a complete quadratic polynomial of $\gamma$ :

$$
P_{i}^{(2)}(\gamma)=P_{0}+\left(\mathbf{P}_{1} \cdot \gamma\right)+\gamma \cdot \mathbf{P}_{2} \gamma
$$

Here, $P_{0}, \mathbf{P}_{1}$, and $\mathbf{P}_{2}$ are scalar, $(n-2)$ - vector, and $(n-2) \times(n-2)$ matrix of coefficients to be determined. Then approximation of the principal component concentration along the boundary of the two-phase domain is introduced in two steps. Firstly, we introduce quadratic approximation for the concentration along the midsurface:

$$
C_{m} \equiv C_{1}^{m}=\frac{1}{2}\left(C_{1}^{l}+C_{1}^{g}\right)=P_{1 m}^{(2)}(\gamma) .
$$

Next, we approximate the square of the differential of phase concentrations as

$$
\left(C_{1}^{g}-C_{1}^{l}\right)^{2}=\left[P_{1 m}^{(2)}(\gamma)\right]^{2} P_{11}^{(2)}(\gamma) .
$$

Therefore, the final expressions are

$$
\begin{gathered}
C^{g} \equiv C_{1}^{g}=P_{1 m}^{(2)}(\gamma)\left(2+\sqrt{P_{11}^{(2)}(\gamma)}\right) \\
C^{l} \equiv C_{1}^{l}=P_{1 m}^{(2)}(\gamma)\left(2-\sqrt{P_{11}^{(2)}(\gamma)}\right) .
\end{gathered}
$$

The sign of the square root is chosen in such a way that the difference $\left(C^{g}-C^{l}\right)>0$ for lightest component. In all cases the coefficients of the approximating polynomials were evaluated using the least mean square procedure. The approximate procedure developed has been tested against available data for a number of reservoir systems. In Fig.3, true and approximated values of the Gibbs energy are compared. Table 1 illustrates the errors of approximation for a number of 4-, and 6 - component systems at a fixed temperature $\mathrm{T}=77^{\circ} \mathrm{C}$, and different pressures.

The values shown in Table 1 are arithmetic mean errors of component concentrations in each of two phases. The errors are less than those usually tolerated in approximating experimentally observed phase behavior by EOS. 


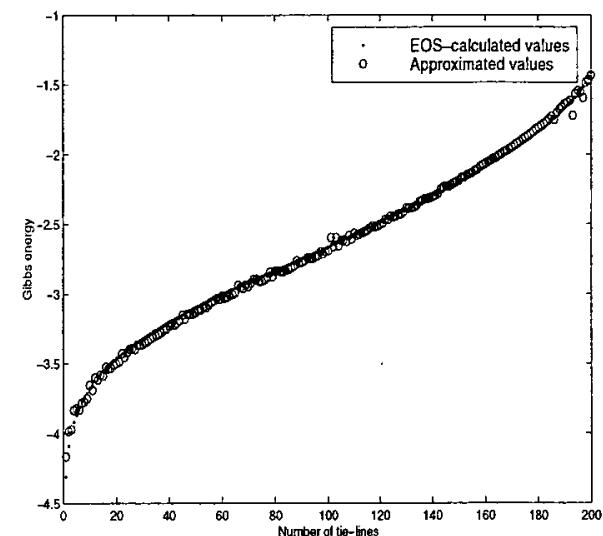

Fig.3 Approximation of Gibbs energy at randomly defined tie-lines for the system $\left[\mathrm{CO}_{2}, C_{1}, C_{4}, C_{10}\right]$ at $T=345^{\circ} \mathrm{K}$ and $\mathrm{P}=120$ Barsa

Table 1. Arithmetic mean approximation errors.

\begin{tabular}{|l|c|c|c|}
\hline System & $\mathrm{P}$ & \multicolumn{2}{|c|}{$\delta_{\text {phase }},(\%)$} \\
\cline { 3 - 4 } & bar & liq. & gas. \\
\hline$\left(\mathrm{CO}_{2}, C_{1}, C_{4}, C_{10}\right)$ & 70 & 0.51 & 0.44 \\
& 100 & 1.14 & 0.96 \\
& 120 & 1.60 & 1.31 \\
& 150 & 1.45 & 1.05 \\
\hline$\left(N_{2}, C_{1}, C_{2}, C_{10}\right)$ & 70 & 0.45 & 0.47 \\
& 100 & 0.84 & 0.83 \\
& 120 & 0.92 & 0.94 \\
& 150 & 0.99 & 0.94 \\
\hline$\left(\mathrm{CO}_{2}, C_{1}, C_{4}, C_{6}, C_{10}\right)$ & 100 & 0.93 & 0.76 \\
& 120 & 1.45 & 1.18 \\
& 150 & 1.68 & 1.34 \\
\hline$\left(\mathrm{CO}_{2}, C_{1}, C_{4}, C_{6}, C_{10}, C_{14}\right)$ & 100 & 0.78 & 0.59 \\
& 120 & 1.33 & 1.02 \\
& 150 & 2.27 & 1.57 \\
\hline$\left(\mathrm{CO}_{2}, C_{1}, C_{2}, C_{4}, C_{6}, C_{10}\right)$ & 100 & 1.16 & 0.97 \\
& 120 & 1.68 & 1.30 \\
& 150 & 1.63 & 1.26 \\
\hline$\left(N_{2}, C_{1}, C_{2}, C_{4}, C_{6}, C_{10}\right)$ & 100 & 1.07 & 0.92 \\
& 120 & 1.32 & 1.08 \\
& 150 & 1.56 & 1.30 \\
\hline
\end{tabular}

Similar calculations can be performed at different $P$ and $T$; in this way, it is easy to find dependence of coefficients of the approximating polynomials on pressure and temperature.

3. Route invariance: numerical experiment. One of main attractions of using the alternative $(\gamma, C)$ formulation of phase equilibria is that according to theoretical predictions in some basic cases the problem splits into two to be solved successively, namely a physicochemical one, formulated entirely in terms of phase equilibria and the tie-line parameter $\gamma$, and hydro- dynamic one, comprising all information related to the flow properties.

More precisely, this statement applies to onedimensional displacement of oil initially at uniform saturation in a semi-infinite reservoir, by gas of a fixed specified composition injected continuously; it is assumed that the pressure differential within the reservoir is negligible, so that displacement occurs at constant pressure. At each time $t$ the displacement is characterized by the composition distribution $\mathbf{C}(x, t)$. The same solution can be expressed as $\gamma(x, t), C(x, t)$ distribution at the reservoir pressure $P$.

The splitting property means that the locus of points $\gamma$ in $\gamma$-space does not depend on $t$ and proves to be the same for different values of injection $C_{\alpha}$ and reservoir $C_{\omega}$ concentrations and different flow-related properties, such as relative permeability functions $k_{r}^{p}$, phase viscosities $\mu^{p}$, provided that the reservoir pressure $P$ and the injection tie-line and reservoir tie-line characterized with respective values of tie-line vector $\gamma_{\alpha}$ and $\gamma_{\omega}$ remain the same. We will refer to the locus as route in tie-line space or just route, and to the above formulated property as route invariance.

Route invariance is illustrated for the ideal case of $1 \mathrm{D}$ displacement for a 5 -component system with constant partition coefficients, in Figs.4. In Fig.4, a concentration distributions as function of spatial (or similarity) coordinate are shown for 3 different phase permeabilities; projection of these solutions into $\gamma$ space is shown in Fig.4,b. All three solutions collapse into a single curve. Notice, that the route invariance property does not apply to route in the concentration space; three different $\mathbf{C}$-routes correspond to three solutions demonstrated.

The route invariance property is formulated under idealized assumptions that are never precisely met for a specific real case. However, it is reasonable to try to represent results of displacement modelling under less restrictive assumptions as routes in the tie-line parameter $\gamma$ space, and check the route invariance property empirically.

Such modelling was performed for a number of cases using compositional simulator Eclipse300 ver.2001. The results expressed in terms of composition vector $\mathbf{C}$ were transformed to tieline parameter variables $\gamma$ assuming constant nom- 


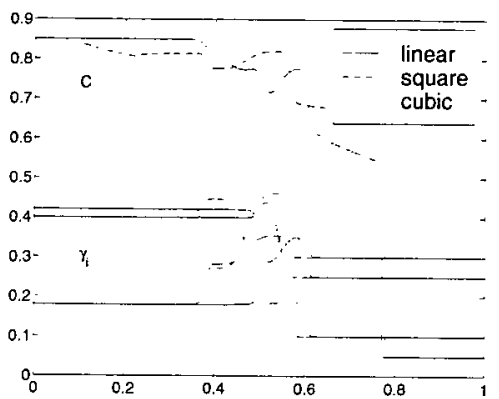

Fig.4,a Distributions of primary variables for 5component system for different fractional flow curves. Power law relative permeabilities with exponents $n_{1}=n_{2}=1,2,3$.

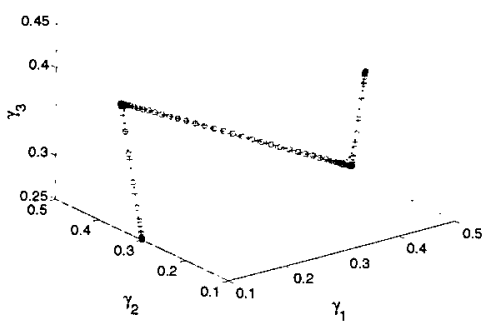

Fig.4,b Route invariance: routes in $\gamma$ - space for three solutions corresponding to different fractional flow curves shown in Fig.4,a.

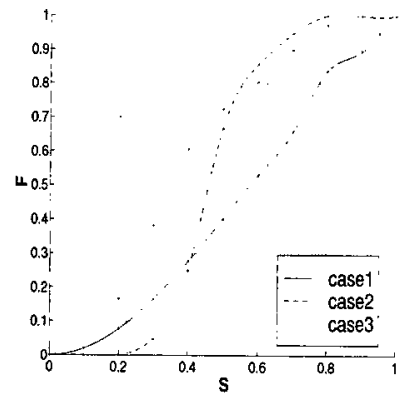

Fig.5,a Fractional flow curves

inal reservoir pressure $P$ equal to the specified reservoir pressure at the beginning of the development.

Figure 5 shows results of modelling linear (1D)displacement for a 4-component system. The reservoir oil was modelled by the typical mixture $\left[C_{1}(19 \%), \mathrm{CO}_{2}(1 \%), C_{4}(40 \%), C_{10}(40 \%)\right]$; injected gas was methane-carbon dioxide mixture of the composition $\left[\mathrm{C}_{1}(20 \%), \mathrm{CO}_{2}(80 \%)\right]$. Three runs were performed using different relative phase permeabilities; respective fractional flow curves are shown in Fig.5,a; pressure differential was of order of 30 per cent of the reservoir pressure.

Figure 5,b shows component distributions for three solutions corresponding to different fractional flow curves. These distributions were used to calculate values of the tie-line vector param-

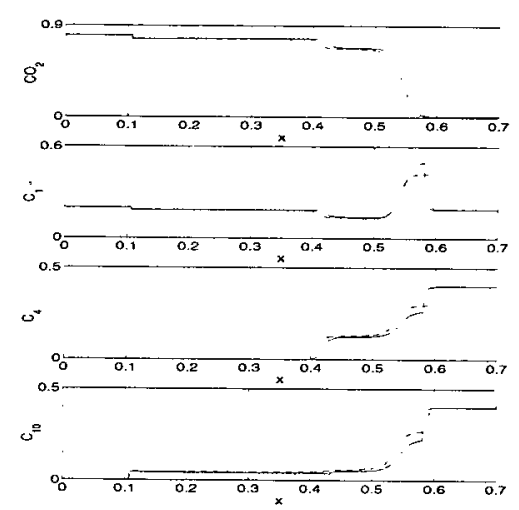

Fig.5,b Component concentration distributions

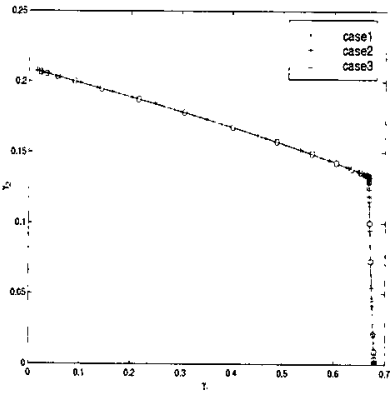

Fig.5,c Route in $\gamma$-space

eter $\left\{\gamma_{1}, \gamma_{2}\right\}$ along the solutions, and plot respective routes in $\gamma$-space; the results are shown in Fig.5,c. The data for different hydrodynamic functions essentially collapse into a single curve (in this case, close to a broken line). Similar results exist for other systems.

The results show that the theory-predicted invariance indeed occurs in the displacement problem.

This led to an effort to apply the projection into tie-line $(\gamma)$ space to more complicated flows with significant pressure variations and/or twodimensional flows. Notice, that there is no theoretical basis for such an effort: the data should not necessarily collapse in a single curve. Nonetheless, the results proved to be rather encouraging. Figure 6 shows results of Eclipse simulations for 2D flow within an element of 5 -spot injection pattern $(40 \times 40$ and $80 \times 80)$ cells in comparison with $1 \mathrm{D}$ solution for the [CO2, C1, C4,C10] system at 120 bars and $345^{\circ} \mathrm{K}$. The oil composition is $[0.01,0.23,0.30,0.46]$; the injected gas composition: $[0.78,0.20,0.01,0.01]$. Despite some scatter, the results definitely tend to single curve.

Essentially, these results show that real displacement follows (if not a single invariant route) a narrow vicinity of the $1 \mathrm{D}$ route in the tie-line 


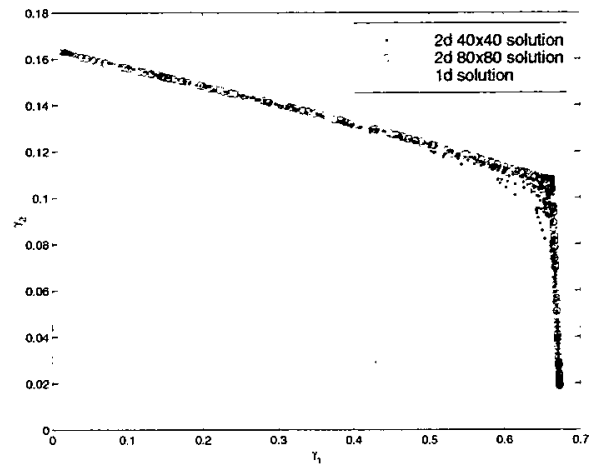

Fig.6 Approximate route invariance in the case of $2 \mathrm{D}$ flow.
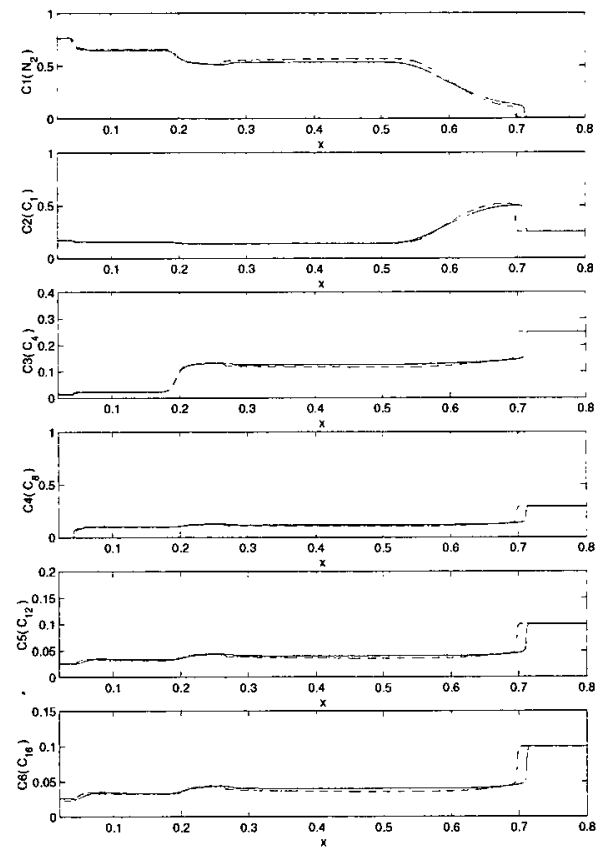

Fig.7 Composition distributions in 1D displacement in 6-component system. Comparison of ECLIPSE, direct solution in natural variables and two-steps results.

space, and this route can be predicted prior to hydrodynamic calculations. The route invariance breaks down at the late stage of displacement, and in the case of significant gravity effects.

There are several means to find the route. The simplest and straightforward with existing software is just to run a compositional simulator for arbitrary chosen relative permeabilities and appropriate initial and boundary conditions, and then to project the solution into the tie-line parameter $(\gamma)$-space. Another technique, theoretically more sound, is first to formulate and solve the auxiliary problem completely formulated in terms of phase equilibria (see [1-7]).

Notice, that the auxiliary ("physicochemical") problem was previously [1-7] formulated, assum- ing constant pressure and temperature, and neglecting the density changes due to mass transfer between the phases. The last limitation, rather confining, can be lifted. It can be shown that for frontal (1D) displacement of oil by gas injection the splitting ("route invariance") property holds also for systems with arbitrary dependence of density on composition (see Section 4).

4. Route invariance in systems with variable density. To prove it, we consider $1 \mathrm{D}$ flow of a mixture described by the set of equations expressing governing conservation laws in terms of the alternative variables $C, \boldsymbol{\gamma}$, molar density $N$ and flow velocity $\mathbf{U}=\{U, 0,0\}$ :

$$
\begin{gathered}
\frac{\partial m N}{\partial t}+\frac{\partial \Phi}{\partial x}=0 \\
\frac{\partial m N C}{\partial t}+\frac{\partial \Phi \Psi(C, \gamma)}{\partial x}=0 \\
\frac{\partial m N\left(A_{i} C+B_{i}\right)}{\partial t}+\frac{\partial \Phi\left(A_{i} \Psi(C, \gamma)+B_{i}\right)}{\partial x}=0 .
\end{gathered}
$$

Here, $A_{i}(\gamma)$ and $B_{i}(\gamma)$ are coefficients in the linear relation between the leading component concentration $C$ and concentration of an intermediate component:

$$
\begin{gathered}
C_{i}=A_{i} C+B_{i} \\
A_{i}=\frac{C_{i}^{l}-C_{i}^{g}}{C^{l}-C^{g}} ; \quad B_{i}=C_{i}^{g}-A_{i} C^{g} .
\end{gathered}
$$

Here,

$$
\begin{gathered}
\Phi=U\left[N^{l} F+N^{g}(1-F)\right] ; \\
\Psi=\frac{N^{l} F C^{l}+N^{g}(1-F) C^{g}}{N^{l} F+N^{g}(1-F)} ; \\
N=N^{l} S+N^{g}(1-S) ;
\end{gathered}
$$

$F(S)$ is fractional flow curve, $N^{l}(\gamma)$ and $N^{g}(\gamma)$ are phase molar densities; $C^{l}(\gamma)$ and $C^{g}(\gamma)$ are values of concentration of the leading component in two phases along given tie-line. Flow is assumed to be isothermal, pressure differential in the reservoir is neglected.

Consider shock conditions for Eqs.(11)-(13):

$$
\begin{gathered}
\Phi^{+}-m N^{+} V=\Phi^{-}-m N^{-} V \\
\Phi^{+} \Psi\left(C^{+}, \gamma^{+}\right)-m N^{+} C^{+} V \\
=\Phi^{-} \Psi\left(C^{-}, \gamma^{-}\right)-m N^{-} C^{-} V ;
\end{gathered}
$$


$=\Phi^{-} \Psi^{-} A_{i}^{-}+\Phi^{-} B_{i}^{-}-m N^{-} C^{-} A_{i}^{-} V-m N^{-} B_{i}^{-} V ;$

Here, $V$ is the shock velocity; superscripts \pm denote limiting values of respective variables ahead and behind the shock wave.

Now, Eqs.(17), (18), and (19) imply

$$
\begin{gathered}
\left(\Phi^{+}-\Phi^{-}\right) \Psi^{-}+\Phi^{+}\left(\Psi^{+}-\Psi^{-}\right) \\
-m N^{+}\left(C^{+}-C^{-}\right) V=0, \\
\Phi^{+} \Psi^{+}\left(A_{i}^{+}-A_{i}^{-}\right)-m N^{+} C^{+} V\left(A_{i}^{+}-A_{i}^{-}\right) \\
+\Phi^{+}\left(B_{i}^{+}-B_{i}^{-}\right)-m N^{+} V\left(B_{i}^{+}-B_{i}^{-}\right)=0 .
\end{gathered}
$$

Therefore the shock wave velocity can be alternatively expressed as:

$$
\begin{gathered}
V=\frac{\Phi^{+}-\Phi^{-}}{m\left(N^{+}-N^{-}\right)}= \\
=\frac{\Phi^{+}\left(\Psi^{+}-\Psi^{-}\right)+\Psi^{-}\left(\Phi^{+}-\Phi^{-}\right)-C^{-}\left(\Phi^{+}-\Phi^{-}\right.}{m N^{+}\left(C^{+}-C^{-}\right)} \\
=\frac{\Phi^{+}\left[\Psi^{+}\left(A_{i}^{+}-A_{i}^{-}\right)+\left(B_{i}^{+}-B_{i}^{-}\right)\right]}{m N^{+}\left[C^{+}\left(A_{i}^{+}-A_{i}^{-}\right)+\left(B_{i}^{+}-B_{i}^{-}\right)\right]} ; \quad \\
V=\frac{\Phi^{+}\left[\Psi^{+}+\frac{\left(B_{i}^{+}-B_{i}^{-}\right)}{\left(A_{i}^{+}-A_{i}^{-}\right)}\right]}{m N^{+}\left[C^{+}+\frac{\left(B_{i}^{+}-B_{i}^{-}\right)}{\left(A_{i}^{+}-A_{i}^{-}\right)}\right]} ; \quad i=1, \ldots, q .
\end{gathered}
$$

Respective characteristic velocities are derived from the shock conditions as the limiting case $Z^{+} \rightarrow Z ; Z^{-} \rightarrow Z ; V \rightarrow \lambda$ :

$$
\begin{gathered}
\lambda=\frac{1}{m} \frac{d \Phi}{d N}= \\
\frac{1}{m N}\left(\frac{d \Phi \Psi}{d C}-\frac{C d \Phi}{d C}\right)= \\
\frac{\Phi}{m N} \frac{\left(\Psi+\frac{d B_{i}}{d A_{i}}\right)}{\left(C+\frac{d B_{i}}{d A_{i}}\right)} ; \quad i=1, \ldots, q .
\end{gathered}
$$

Equations (23) and (24) are crucially important. Essentially they show that, provided the tie-line parameter $\gamma$ does not remain constant along a similarity solution, respective variations $d A_{i}$ and $d B_{i}$ or $\delta A_{i}$ and $\delta B_{i}$ should be correlated in the sense that relations

$$
d B_{i}=\eta d A_{i} ; \text { or } \delta B_{i}=\eta \delta A_{i},
$$

hold true simultaneously for all $i=1, \ldots q$. However, this means that $A_{i}$ and $B_{i}$ satisfy a Riemann problem for the auxiliary system of conservation laws:

$$
\frac{\partial \mathbf{A}}{\partial t}+\frac{\partial \mathbf{B}}{\partial y}=0 ; \quad \gamma( \pm \infty, 0)=\gamma^{ \pm},
$$
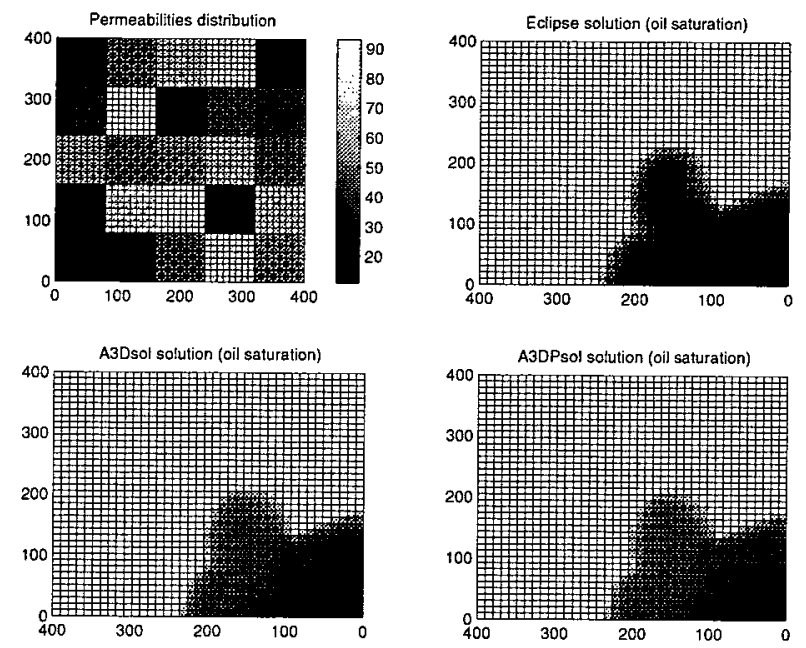

Fig. $8 \mathrm{Oil}$ displacement by $\mathrm{CO}_{2}$ injection in a five-spot element with random permeability distribution; top - the permeability map and ECLIPSE simulation; right - alternative simulation at $P=$ const (left) and alternative simulation at $P \neq$ const (right)

in exactly the same way as for the constant phase densities case treated previously [1-7]. This implies that fundamental principles, such as splitting of the displacement problem into physicochemical and hydrodynamic ones and route invariance with respect to variation of hydrodynamic variables, remain valid for the variable density case as well. Notice, that Eqs.(23) and (24) are nothing else than condition of intersection of each two consecutive tie-lines along the route in composition space, the property, explicitly stated by Orr et al [8-10]. Therefore the route to be found can be defined as an ordered sequence of tie-lines starting from the injection tie-line and ending at the initial-state tie-line in which each two adjacent tie-lines have a common point. (Of course, the set of tie line can be finite, infinite countable, or uncountable).

\section{Alternative compositional simula-} tor. Among different applications of the "natural compositional coordinates", one of options is just to use them in direct compositional simulations. In this case, the conservation laws are first expressed in terms of the variables $C, \gamma, P$, and then the flow equations are solved by an analogue of IMPES technique. Some results are shown in Fig.8-9.

\section{Conclusion}

Main results of this study are:

1. Development of an effective technique of description of phase equilibria in multicomponent systems in terms of natural variables, namely 


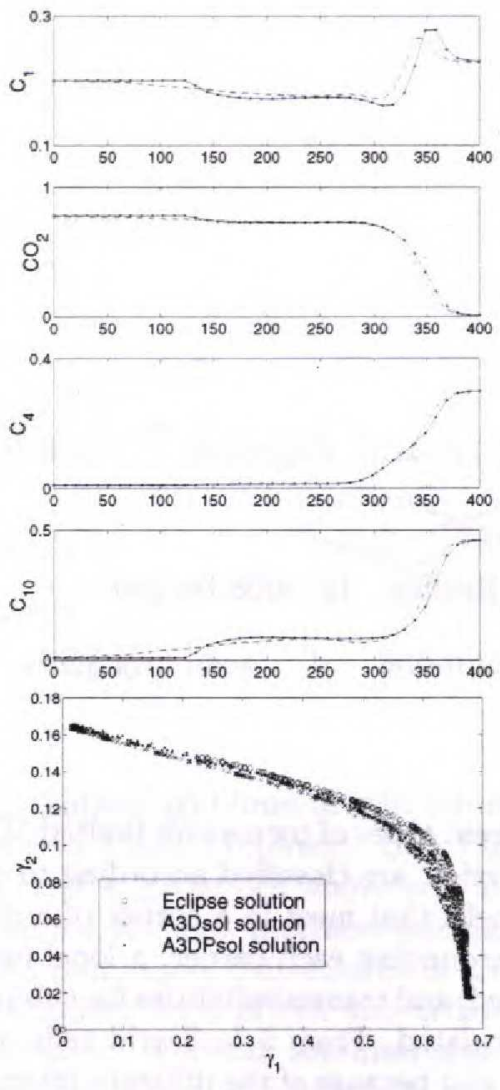

Fig.9 Oil displacement by $\mathrm{CO}_{2}$ injection in a five-spot element with random permeability distribution:

a: concentrations distribution along principal streamline; $\mathbf{b}$ : routes in $\gamma$-space.

the main component concentration and tie-line vector-parameter.

2. Demonstration of route invariance property for realistic $1 \mathrm{D}$ and $2 \mathrm{D}$ flows simulated by commonly used compositional simulators.

3. Extension of the route invariance property on flows with composition-dependent density.

4. Development of a prototype compositional simulator based on natural coordinates in composition space.

Whatever be further development, it looks rather plausible, that explicit using the route invariance property and/or natural coordinates in component space for description of phase equilibrium can lead to more concise and transparent predictions of multicomponent flows.

6. Bibliographical notes. As stated in [1-7], this study was initiated under impression of activity of Prof.F.Orr,Jr and his colleagues at Stanford University [8-10]. It proved, that a similar approach had been suggested and partially realized previously by P.Bedrikovetsky and M.Chumak. [11,12]. In particular, their state- ments relative the constant partition case are very close to those considered in more detail in [1-7]. Splitting of the displacement problem into physicochemical and hydrodynamical parts was discussed by A.Brusilovsky and A.Zazovsky [13] in a different framework. The reader is invited to consult these references to compare approaches used.

Acknowledgements. Financial support of Schlumberger, access to Eclipse compositional simulator and many useful discussions at Schlumberger Moscow Research and Schlumberger - Geoquest (Abingdon, UK), as well as discussions with A.Barmin and A.Brusilovsky are gratefully acknowledged.

\section{References}

1. Entov, V.M., in: Proc. of the Conference "Porous Media, Physics, Models, Simulations," Moscow, 19-21 Nov., 1997, A.N.Dmitrievsky, M.B.Panfilov, eds. Singapore, 2000.

2. Entov, V.M., in: Proc. Intern. Conf. "Modern Approaches to Flows through Porous Media", Moscow, Sept.6-8, 1999, 14-16.

3. Voskov, D.V., and Entov V.M., in: Proc. Intern. Conf. "Modern Approaches to Flows through Porous Media", Moscow, Sept.6-8, 1999, II-32-II-34.

4. Voskov D.V., and Entov V.M., Izv. RAN, Mekh. Zhidk. Gaza [Fluid Dynamics], 2001, N2, 112-121.

5. Entov V.M., and Voskov D.V. Proc. Intern. Conf. on Multiphase Systems, ICMS'2000, Ufa, Russia, June 15-17, 2000, 401-408.

6. Entov V.M., and Voskov D.V., 2000. Proc of 7th European Conference on the Mathematics of Oil Recovery, Baveno, Italy, 5-8 September 2000.

7. Voskov D.V., PhD Thesis, 2002, Gubkin Russian State Oil and Gas U., Moscow, Russia.

8. Monroe, W.W., Silva, M.K., Larsen, L.L., and Orr, F.M.,Jr Soc.Petr.Eng. Res.Eng. 1990, 5, 423432.

9. Orr F.M. Jr., Johns, R.T., and Dindoruk, B., Soc. Petr. Eng., Res. Eng. 1993, 8, 135-142.

10. Johns R.T., Orr F.M. Jr., and Dindoruk B., Soc. Petr. Engrs. Advanced Technology Series, 1993, 1(2), 7-16.

11. Bedrikovetskii, P.G. Chumak, M.L. Doklady Akad. Nauk SSSR, 1992, 322,(4-6), 668-73.

12. Bedrikovetsky P.G., Chumak M.L., 1992, Proc of 3rd European Conference on the Mathematics of Oil Recovery. Delft, The Netherlands.

13. Brusilovsky A.I., Zazovsky A.F., 1991, SPE Paper 22638. 